\title{
El Personal Learning Environment en el proceso de aprendizaje de estudiantes de nivelación: un caso en la Universidad de Guayaquil/Ecuador
}

\section{A Personal Learning Environment for students in a leveling process: a case study at the University of Guayaquil/Ecuador}

\author{
Oswaldo Aráuz Arroyo ${ }^{1}$, Annabelle Estefanía Figueroa Lizarzaburu ${ }^{1}$ \\ ${ }^{1}$ Universidad Casa Grande, UCG, Guayaquil, Equador
}

Correo a/Mail to: Oswaldo Aráuz Arroyo oswaldo_arauz@hotmail.com

Recibido/Submitted: 26 Oct. 2015; Aceptado/Approved: 27 Nov. 2015

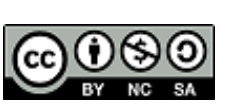

\begin{abstract}
Copyright (C) 2015 Aráuz Arroyo \& Figueroa Lizarzaburu. Todo el contenido de la revista (incluyendo instrucciones, política editorial y modelos) está bajo una licencia Creative Commons Atribución-NoComercial-Compartirlgual 3.0 No Adaptada. Siendo publicados por esta revista, artículos son de libre uso en ambientes educativos, de investigación y no comerciales, con atribución de autoría obligatoria. Más información en http://ojs.c3sl.ufpr.br/ojs2/index.php/atoz/about/submissions\#copyrightNotice.
\end{abstract}

\begin{abstract}
Resumen
Introducción: El objetivo de la presente investigación es identificar las ventajas pedagógicas, comunicativas y de aprendizaje en la implementación de un modelo de formación innovador en los estudiantes de nivelación de la Universidad de Guayaquil, o sea, la creación y desarrollo de un personal learning environment (PLE) como proyecto de aula.

Método: Para el presente caso de estudio se consideró una estrategia metodológica mixta y se realizó en cuatro etapas: (a) Socialización del concepto del PLE, (b) Desarrollo del PLE por parte del alumnado y retroalimentación entre pares en un blog creado para este fin, (c) Evaluación del PLE desarrollado por los estudiantes y (d) Encuesta para medir el nivel de satisfacción y motivación obtenido por los educandos.

Resultados: Los resultados obtenidos permiten percibir el alto grado de satisfacción, compromiso e interés que los estudiantes desarrollaron al implementar el PLE como proyecto de aula, llegando a considerar esta estrategia educativa como una práctica innovadora y que les permitió descubrir nuevas habilidades y elevar sus niveles de interacción y colaboración entre pares.

Conclusiones: Se concluye que el desarrollo del PLE como proyecto de aula derivó en una experiencia motivante y satisfactoria para los estudiantes, logrando enriquecer sus conocimientos sobre las TIC, desarrollar su creatividad y contribuir de forma importante en su proceso de aprendizaje.
\end{abstract}

Palabras clave: Entornos Personales de Aprendizaje (PLE); Estilos de aprendizaje; Innovación en educación

\begin{abstract}
Introduction: The objective of this research is to identify the educational, communicative and learning advantages in implementing an innovative model of training for leveling students at the University of Guayaquil, that is, the creation and development of a personal learning environment (PLE) as a classroom project.

Method: For this case study it was considered a mixed approach conducted in four stages: (a) Socialization of PLE concept, (b) Development of PLE by students and peer feedback in a blog created for this purpose, (c) Evaluation of PLE developed by students and (d) Survey to measure the level of satisfaction and motivation reached by students.

Results: The results allowed the perception of high levels of satisfaction, commitment and interest developed by the students when implementing the PLE as a classroom project. It was possible to consider this educational strategy as an innovative practice that enabled them to discover new skills and enhancing their levels of interaction and collaboration among peers.

Conclusions: The development of PLE as a classroom project resulted in a motivating and satisfying experience for students, enriching their knowledge about ICT, as well as, developing their creativity and contributing significantly to their learning process.
\end{abstract}

Keywords: Personal Learning Environments (PLE); Learning styles; Innovation in education

\section{INTRODUCCIÓN}

Actualmente los acelerados avances tecnológicos que se han convertido en el diario vivir de las personas también se han comenzado a introducir en las instituciones educativas a pesar de la resistencia de las mismas para integrarlos (Blázquez, 2001). Los seres humanos se encuentran en una época en la que la información los rodea y pueden acceder a ella de una forma sencilla; sólo es necesario un click y la obtienen en el formato, en el lugar y a la hora que decidan. En palabras de Adell y Castañeda (2013, p. 12)

...eso significa que las experiencias, intercambios, actividades a las que nos ha acercado el uso de las tecnologías ha multiplicado, diversificado y personalizado de manera extraordinaria nuestro entorno para aprender, al punto que los entornos de aprendizaje centralizados y comunes a todos nos parecen insuficientes y empobrecedores.

Todo lo anteriormente mencionado fundamenta la importancia que está tomando el nuevo concepto del personal learning environment (PLE) en el terreno educativo. Por lo que la definición actual del PLE es “....el conjunto de herramientas, fuentes de información, conexiones y actividades que cada persona utiliza de forma asidua para 
aprender” (Adell \& Castañeda, 2010, p. 23). Esta nueva visión de PLE plantea diferentes aspectos relacionados a la forma de aprendizaje en la sociedad del conocimiento, y cómo los saberes se han transformado en información que fluye, no solamente en las universidades y otras instituciones formales, dejando estos sitios de ser los monopolizadores del conocimiento y permitiendo que nuestro abanico de posibilidades se amplíe a los contextos no formales e informales con la combinación de lo presencial y lo virtual (Cabero Almenara, 2014).

Todos los individuos poseen un entorno personal de aprendizaje donde obtienen conocimiento, aunque no estén conscientes de ello; al existir una interacción familiar, escolar o de amigos, se puede considerar que ya están dentro de uno sin siquiera haberlo notado. Años atrás la adquisición de conocimiento era limitada a un modelo centrado en un profesor-experto que brindaba los saberes (que según sus criterios los estudiantes debían conocer) y eso era más que suficiente. Además esta información era trascendental y no cambiaba con el tiempo. Pero el contexto actual es completamente diferente. Con la aparición de las TIC que facilitan el acceso a la información todo ha cambiado y

\begin{abstract}
ahora podemos acceder de forma rápida y sencilla a toda la información que constituía en otros momentos el grueso de la educación escolar (los contenidos) y además podemos comentarla, recrearla y debatirla con otras personas. Casi todo lo que nos pueda interesar está a distancia de un clic. (Adell \& Castañeda, 2013, p. 12)
\end{abstract}

Según algunos autores, el contexto en que el ser humano se desenvuelve en la actualidad se denomina una web socialmente conectada, que permite a los usuarios potenciar la idea de mayor contribución frente a mayor consumo (Anderson, 2007). Es donde la mayor parte de las herramientas y recursos disponibles (wikis, blogs, YouTube, redes sociales, marcadores, etc.) se centran en posibilitar y promover en el usuario la creación y la distribución de contenidos, a través de la participación, la interacción y la colaboración entre todos, de ahí que se apele a la etiqueta de "social" (Llorente Cejudo, 2013, p. 41). Debido a esto, el entorno/espacio personal de aprendizaje (PLE) también ha cambiado en condiciones tanto social y cultural, y es únicamente restringido por las limitaciones tecnológicas que tengan las personas. Este desarrollo de la tecnología lo potencializa y hace que la información adquirida y creada por cada persona sea aprovechada frecuentemente y que sirva para engrandecer la forma en la que aprendemos en la actual sociedad del conocimiento.

Pero existen algunos inconvenientes para la aplicación del PLE como herramienta de aprendizaje, debido a que según Ordóñez (2006) en el ámbito universitario los profesores tienen una tendencia a mostrarse extrañados e incrédulos con la tecnología. Para ellos la transmisión de conocimiento sigue siendo el propósito principal de la educación, aunque estemos en una época donde la información está al alcance de todos por medios diversos y eficientes. Donde ella misma cambia cada día, donde el volumen producido hace totalmente imposible 'cubrirla' y debería ser la consideración crítica autónoma de esa información, por parte de quien aprende, lo que realmente se debe perseguir pedagógicamente. Sin embargo, vale acotar que no todos los docentes poseen este pensamiento tradicionalista y algunos si utilizan las TIC en su vida diaria tanto personal y como ayuda en la preparación de sus clases (Hinojo Lucena, Fernández Martín, \& Aznar Díaz, 2002), a pesar de que su uso como herramienta de aprendizaje en el aula es limitado, debido a la dificultad que se presenta al desconocer cómo integrar la clase a las nuevas herramientas tecnológicas y a las limitaciones de tipo técnico y económico; ya que no en todos los centros educativos se dispone de la última tecnología (Carneiro, Toscano, \& Díaz, 2009).

El reto consiste en incentivar un cambio que no solo involucre la incorporación de las TIC, sino una transformación en la actitud de los docentes, donde se permita que el estudiante pueda formar parte en el proceso de enseñanza y aprendizaje a través de una educación autónoma, en la que el profesor se encuentre capacitado en diferentes habilidades y destrezas para establecer transformaciones en lo que al diseño y a la planificación de saberes se refiere (Llorente Cejudo, 2013). Por lo que se identifica la necesidad de crear canales efectivos de interacción entre docentes y alumnos, con el objeto de intercambiar experiencias y dar piso a una comunidad académica también llamada sociedad del conocimiento, que abra espacios de análisis, reflexión y aprendizaje de un sinfín de temas.

En Ecuador, la utilización de las Tecnologías de la Información y Comunicación (TIC) está creciendo y las experiencias transformadoras que se están creando apoyan en forma beneficiosa a todos los gestores del aprendizaje. Una de ellas es el caso del internet que, según datos del Instituto Nacional de Estadística y Censos de Ecuador (2012), refleja que: "el 28,3\% de los hogares a nivel nacional tienen acceso a internet, 16,5 puntos más que en el 2010. En el área urbana el crecimiento es de 20,3 puntos, mientras que en la rural de 7,8 puntos”. Sin embargo, el uso de los PLE es limitado quizás por el desconocimiento de las bondades que este puede ofrecer en el aprendizaje, debido a que no muchos lo consideran como un modelo de formación virtual con mucho potencial para el aprendizaje autónomo de los estudiantes, en el que tanto docentes como alumnos somos los que decidimos qué y de quién aprender (Adell \& Castañeda, 2013). Al crear y desarrollar el PLE a gusto de cada individuo, se está abriendo un horizonte a una nueva y enriquecedora forma de educación, ya que el uso de las TIC que conforman el PLE facilitan que uno no se limite a ser receptor del conocimiento, sino que se transforme en actor del mismo.

A partir de esas consideraciones el objetivo general de la investigación es de identificar las ventajas pedagógicas, 
comunicativas y de aprendizaje en la implementación de un modelo de formación innovador en los estudiantes: Creación y desarrollo de un PLE como proyecto de aula. Los objetivos específicos son los de evaluar el grado de importancia que los estudiantes dan al PLE en su futura vida profesional y cuantificar el nivel de motivación que genera esta experiencia educativa en ellos.

\section{PROCEDIMIENTOS METODOLÓGICOS}

Al tener un diálogo ameno y abierto con los estudiantes se llegó a comprender que el uso de las TIC es parte primordial en su desarrollo educativo y personal. La táctica pensada fue dejar a un lado la tradicional forma de presentar el proyecto final áulico - que consistía en la investigación y posterior redacción de un informe impreso para el profesor y una presentación muchas veces monótona para sus compañeros - planteándoles la creación de su propio entorno virtual de aprendizaje PLE. Está propuesta tuvo una buena acogida por todos, ya que lo tomaron como un reto, y al hacerlo en forma virtual vieron la posibilidad de incorporar a su aprendizaje algo que para ellos les es familiar, lo que refiere Bruner (1987, p. 88) como "encontrar la conexión con algo que ya conocen".

Para el presente caso de estudio se consideró una estrategia metodológica mixta que según Pereira Pérez (2011, p. 19) aclara que “... los métodos cuantitativos se utilizan en una etapa o fase de la investigación y los cualitativos en otra”. Esto se debe a que la propuesta de motivar la creación de un PLE posee factores que no se alcanzarían a medir en una forma únicamente cuantitativa, ya que gran parte de estos datos son de carácter cultural, conductual, demográfico y entre otras que son propias de cada estudiante. Por lo que es necesario la cuantificación de datos recolectados mediante una encuesta, además de complementar la información con la técnica de observación y análisis de los proyectos que van a ser parte de los datos cualitativos.

Para esta investigación se han tomado dos grupos de alumnos correspondientes a los cursos de nivelación de la SNNA de la Universidad de Guayaquil/Ecuador, período Marzo - Agosto 2015, conformando por 35 estudiantes de la Facultad de Ciencias Naturales Paralelo A y por 32 estudiantes de la Facultad de Filosofía Paralelo A7. La investigación se desarrolló como parte de un proyecto de aula que se solicita a todos los docentes de nivelación como parte del plan de clase. Considerando la realidad actual en la que esos alumnos se desenvuelven - rodeados de un entorno tecnológico lleno de herramientas de aprendizaje y a su vez de elementos distractores que afectan el rendimiento y generan poco compromiso en sus estudios - el profesor percibió la necesidad imperante de crear estrategias interactivas que lleguen a motivarlos y a involucrarlos activamente en su formación. Piaget pensaba que "el principal objetivo de la educación debería consistir en ayudar a los estudiantes a aprender cómo aprender" (Woolfolk, 2006, p. 40).

El estudio se realizó en cuatro etapas: (a) Socialización del concepto del PLE; (b) Desarrollo del PLE por parte del alumnado y retroalimentación entre pares en un blog creado para este fin; (c) Evaluación del PLE desarrollado por los estudiantes; y, (d) Encuesta para medir el nivel de satisfacción y motivación obtenido por los educandos.

En primer lugar, se invitó a todos los estudiantes a que se familiaricen con la estructura de un PLE tomando como ejemplo el del docente, con lo que pudieron observar sus componentes y como se puede mezclar lo educativo con lo personal, teniendo una interacción con otros mediante blogs o correos electrónicos para así generar un aprendizaje colaborativo. En segundo lugar, se propuso a los alumnos la elaboración de su propio PLE dentro de una Webquest definida por Argote, Palomo, Sánchez, y Ruiz (n.d.) como una actividad en la que se trata de buscar información en forma guiada, en la que la mayor parte va a ser extraída del Internet y es diseñada para optimizar el trabajo de los alumnos, centrándolos en el procesamiento de datos en lugar de un proceso solo de búsqueda de los mismos. Esta actividad formaba parte del proyecto de aula y para la valoración objetiva de los PLEs se desarrolló una rúbrica (Apéndice A) que fue aplicada por el docente y los alumnos a todos los PLEs presentados.

Para finalizar la investigación y lograr cuantificar el grado de satisfacción y aprendizaje que obtuvieron los participantes en este proceso se procedió a diseñar una encuesta descriptiva de preguntas cerradas vía online, la cual fue enviada a los estudiantes para su contestación.

\section{RESULTADOS Y DISCUSIÓN}

Los resultados obtenidos a través de la metodología cualitativa; utilizando la herramienta de observación, permitieron percibir el alto grado de satisfacción e interés que los estudiantes desarrollaron al implementar el PLE como proyecto áulico. Se pudo además observar los elevados niveles de interacción y colaboración entre pares propiciados por la socialización de los proyectos en el blog, y el compromiso de los estudiantes en realizar trabajos que denotaron calidad y esfuerzo a pesar de la falta de recursos y/o facilidades informáticas. Todo esto sustentado en gran medida con los recursos proporcionados por la webquest desarrollada para este proyecto.

En lo que respecta a los datos obtenidos a través de la investigación cuantitativa, se obtuvo que del total de 67 alumnos que participaron, un $75 \%$ respondió a la encuesta. A continuación se presentan los resultados más 
relevantes que permitirán obtener un análisis más profundo de la problemática. Para empezar la Figura 1.a indica que el $74 \%$ de los encuestados obtuvo el conocimiento de la creación de un PLE gracias a la ayuda del docente.

En la Figura 1.b se denota que un $64 \%$ de los encuestados está de acuerdo con la importancia que actualmente tiene el desarrollo de un PLE para su crecimiento académico, lo que refleja el interés que los jóvenes tienen en ser gestores de su conocimiento e incorporar las tecnologías de la información y comunicación a su diario vivir.

En la Figura 1.c se puede observar que la estrategia planteada de desarrollar un PLE como proyecto áulico generó un impacto positivo y una experiencia motivadora en más del $60 \%$ del alumnado; así como el descubrimiento de nuevas habilidades que ellos desconocían poseer.

La Figura 1.d refleja que un 64\% de los estudiantes encontraron como una práctica innovadora el hecho de cambiar la tradicional forma de desarrollar los proyectos áulicos.

En la Figura 1.e se evidencia que el 78\% de los encuestados considera que el PLE les ayudará en sus futuros procesos de aprendizaje profesionales y académicos. La alta aceptación de esta herramienta motivará a los alumnos a que su uso no quede limitado a un aula de clase y que este conocimiento adquirido trascienda a otros campos de su vida.
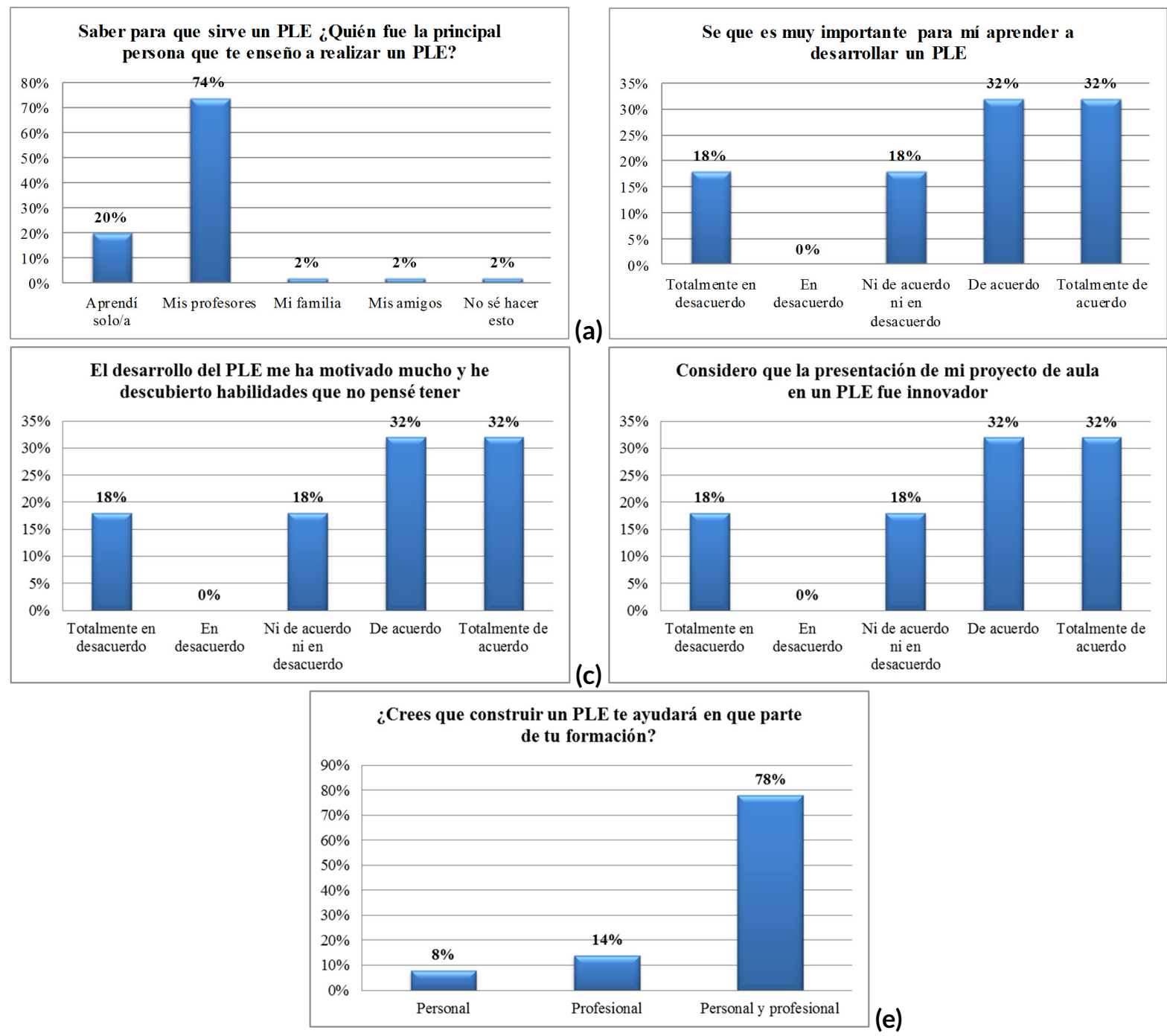

Figura 1. (a) ¿Quién fue la principal persona que te enseño a realizar un PLE?. (b) Importancia de desarrollar un PLE para el aprendizaje. (c) Motivación en los estudiantes al desarrollar su PLE. (d) Consideración de la innovación en los estudiantes al desarrollar su PLE. (e) ¿Crees que construir un PLE te ayudará en que parte de tu formación?.

Fuente: los autores.

Además, la encuesta aplicada a la muestra incluyó preguntas acerca de frecuencias de uso, y otros aspectos relacionados a las TIC. Del análisis de estos resultados se encontró que el 94,3\% tiene acceso a un computador y que el 84,9\% de ellos sólo tiene acceso al internet cuando acuden a sitios donde se prestan estos servicios (cybers), lo que demuestra que a pesar de no poseer estos recursos en casa, existió el compromiso por parte de los estudiantes para cumplir con el requerimiento del proyecto de aula.

Las Figuras 2.a e .b muestran los PLEs que obtuvieron una mejor valoración por parte del docente y sus compañeros. 


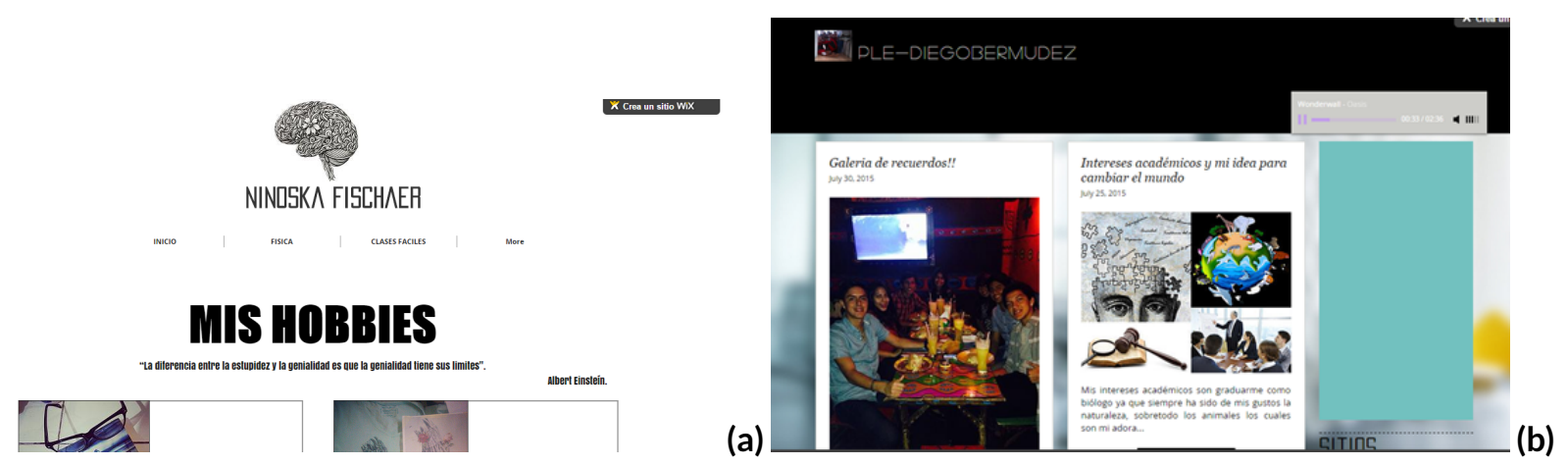

Figura 2. (a) Ejemplo de PLE de estudiante de nivelación de la Universidad de Guayaquil/Ecuador. (b) Ejemplo de PLE de estudiante de nivelación de la Universidad de Guayaquil/Ecuador.

Fuente: (a) Captura de pantalla de PLE, autor Ninoska Fischaer, alumna Fac. de Ciencias Naturales, recuperado de http://ninoskafischer22.wix.com/ple-ninoskafischerg en 2/09/2015. (b) Captura de pantalla de PLE, autor Diego Bermúdez, alumno Fac. de Ciencias Naturales, recuperado de http://diegobenjamin12.wix.com/ple-diegobermude en 2/09/2015.

Como limitante en la implementación de la metodología se puede mencionar el acceso al uso de las facilidades comunicativas (proyector y acceso a internet) por parte de ambas facultades. Al momento de la explicación de los requerimientos del trabajo de aula, una de las facultades no brindo el recurso tecnológico necesario lo que forzó el investigador a dar una breve explicación con el apoyo de un documento en el cual se adjuntaron imágenes de pantalla capturadas para orientar a los estudiantes.

Para lograr la extrapolación de esta experiencia a otros procesos educativos de la Universidad de Guayaquil se recomienda la incorporación de las TIC en las aulas como apoyo a la gestión del docente y como herramienta base para la correcta ejecución de esta estrategia de aprendizaje.

\section{CONCLUSIONES}

El desarrollo del PLE como proyecto de aula derivó en las ventajas pedagógicas, comunicativas y de aprendizaje esperadas. Los estudiantes en su mayoría coincidieron en encontrar este modelo de formación- el cual fue aplicado con una metodología de aprender haciendo - como una experiencia motivadora e innovadora, que enriqueció sus conocimientos sobre las TIC y permitió su aplicación inmediata en sus proyectos áulicos. Además de generar una colaboración directa entre todos los gestores del conocimiento (docente y alumnos) y promover el desarrollo de nuevas habilidades de comunicación, como la retroalimentación entre pares.

Al finalizar el curso de nivelación se procedió a realizar la revisión de los PLEs de los estudiantes para verificar resultados, y el grado de satisfacción obtenido por el docente fue excepcional e inesperado. A pesar de no poseer recursos económicos y fácil acceso a la tecnología debido al estrato socioeconómico al que pertenecen, los estudiantes en su gran mayoría hicieron trabajos que denotaron mucho empeño, creatividad y compromiso, superando las expectativas del docente al comienzo del curso.

La flexibilización de los conocimientos adquiridos al realizar el PLE como proyecto áulico fue considerado por el alumnado como una contribución relevante en su proceso de aprendizaje y consideran que este conocimiento puede ser aplicable en su futuro académico y profesional. Sin embargo, al analizar los resultados obtenidos se ve la necesidad de contar con los recursos tecnológicos apropiados para la inducción inicial, además de derivar un poco más de tiempo para la revisión en aula de los proyectos y con este fin mejorar la experiencia de los alumnos.

Esta experiencia ha contribuido de forma positiva tanto a los estudiantes como a la plana docente y servirá de base para que en los próximos cursos de nivelación se posibilite la consecución de proyectos similares. Los modelos de PLEs creados por los alumnos se utilizarán como ejemplo para nuevos participantes de procesos de nivelación, siendo esta una forma de motivarlos e incentivarlos a la elaboración de sus propios entornos personales. Además, los comentarios positivos obtenidos a raíz de esta investigación, promovieron a las autoridades de la Universidad a organizar una capacitación sobre entornos personales y aprendizaje virtual para todos los docentes involucrados en el proceso de nivelación, con el fin de que ellos también apliquen esta estrategia con sus alumnos. 


\section{REFERENCIAS}

Adell, J., \& Castañeda, L. (2010). Los entornos personales de aprendizaje (PLEs): una nueva manera de entender al aprendizaje. Roma: Marfil.

Adell, J., \& Castañeda, L. (2013). Entornos personales de aprendizaje: claves para el ecosistema educativo en red. Alcoy: Marfil.

Anderson, F. (2007). ¿Qué es la Web 2.0? Las ideas, tecnologías e implicaciones para la educación. JISC Technology and Standards Watch. Descargado de http://www.jisc.ac.uk/ media/documents/techwatch/tsw0701b.pdf

Argote, J., Palomo, R., Sánchez, J., \& Ruiz, J. (s.f.). Webquest: Un recurso educativo para su uso en el aula. Málaga: Universidad de Málaga. Descargado de http://tecnologiaedu.uma.es/ index.php/materiales/23-curso-de-webquest-un-recurso -educativo-para-su-uso-en-el-aula

Blázquez, F. (2001). Sociedad de la información y educación. Mérida: Junta de Extremadura.

Bruner, J. (1987). La importancia de la educación. Barcelona: Paidós.

Cabero Almenara, J. (2014). Creación de entornos personales de aprendizaje como recurso para la formación: El Proyecto Dipro 2.0. Edute Revista Electrónica de Tecnología Educativa(47), 1-16. Descargado de http://www.edutec.es/revista/ index.php/edutec-e/article/view/80/pdf_1

Carneiro, R., Toscano, J., \& Díaz, T. (2009). Los desafíos de las TIC para el cambio educativo. Madrid: Fundación Santillana.

Hinojo Lucena, F. J., Fernández Martín, F. D., \& Aznar Díaz, I. (2002). Las actitudes de los docentes hacia la formación en tecnologías de la información y comunicación (TIC) aplicadas a la educación. Contextos Educativos: Revista de educación(5), 253-270. Descargado de http://dialnet.unirioja.es/servlet/ articulo? codigo $=498346$

Instituto Nacional de Estadística y Censos de Ecuador. (2012). Tecnologías de la información y comunicaciones (TICs). Descargado de http://www.inec.gob.ec/sitio_tics2012/presentacion .pdf

Llorente Cejudo, M. C. (2013). Assessing personal learning environments (ples). an expert evaluation. Journal of New Approaches in Educational Research, 2(1), 39-44. doi: 10 .7821/naer.2.1.39-44

Ordóñez, C. L. (2006). Pensar pedagógicamente, de nuevo, desde el constructivismo. Revista Ciencias de la Salud, 4(Especial), 14-23. Descargado de http://revistas.urosario .edu.co/index.php/revsalud/article/view/539

Pereira Pérez, Z. (2011, Enero-Junio). Los diseños de método mixto en la investigación en educación: Una experiencia concreta. Revista Electrónica Educare, 15(1), 15-29. Descargado de http://www.redalyc.org/pdf/1941/ 194118804003.pdf

Woolfolk, A. (2006). Psicología educativa. México: Pearson.

Cómo citar este shortpaper (APA):

Aráuz Arroyo, O. \& Figueroa Lizarzaburu, A. E. (2015). El Personal Learning Environment en el proceso de aprendizaje de estudiantes de nivelación: un caso en la Universidad de Guayaquil/Ecuador. AtoZ: novas práticas em informação e conhecimento, 4(2), 95 - 102. Descargado de: http://dx.doi.org/10.5380/atoz.v4i2.43680 


\section{APÉNDICE A - RÚBRICA DE EVALUACIÓN DE PLES}

(33.33\%) La información contenida es fácil de interpretar

Excelente Bueno Satisfactorio Deficiente

$(100.00 \%)(75.00 \%)(50.00 \%) \quad(25.00 \%)$

(22.22\%) Apariencia del PLE

$\begin{array}{llll}\text { Excelente } & \text { Bueno } & \text { Satisfactorio } & \text { Deficiente } \\ (100.00 \%) & (75.00 \%) & (50.00 \%) & (25.00 \%)\end{array}$

$(21.88 \%)$ Uso de paleta de colores que son armoniosos entre si.

$(21.88 \%)$ El texto es claro y legible con facilidad.

(15.63\%) Las imágenes y vídeos contenidos son de buena calidad.

(9.38\%) Las herramientas y recursos son actuales.

(9.38\%) Las herramientas y recursos son confiables.

$(21.88 \%)$ Presencia de faltas ortográficas y de redacción $\left(^{*}\right)$

\section{(32.00\%) Carácter Educativo del PLE}

(41.18\%) Incentiva a la educación

$$
\begin{array}{llll}
\text { Excelente } & \text { Bueno } & \text { Satisfactorio } & \text { Deficiente } \\
(100.00 \%) & (75.00 \%) & (50.00 \%) & (25.00 \%)
\end{array}
$$

$(45.00 \%)$ Contenidos que promuevan al aprendizaje. $\left({ }^{*}\right)$

$(40.00 \%)$ Herramientas que permitan la retroalimentación y debate de los contenidos

(15.00\%) Presencia de herramientas o contenidos que innove en nuevas formas de aprender

(58.82\%) Incentiva a conocer al propietario

$\begin{array}{llll}\text { Excelente } & \text { Bueno } & \text { Satisfactorio } & \text { Deficiente } \\ (100.00 \%) & (75.00 \%) & (50.00 \%) & (25.00 \%)\end{array}$

(50.00\%) Da formas claras de conocer los interesas y gustos del dueño

$(50.00 \%)$ Presenta herramientas que permitan una interacción social con el dueño $\left({ }^{*}\right)$

$\left(^{*}\right)$ Esta competencia/evidencia es necesaria para aprobar 


\section{Evaluación de competencias en la elaboración de un PLE}

\section{(40.00\%) Estructura del PLE}

$\mathbf{( 5 3 . 3 3 \% )}$ Orden y accesibilidad

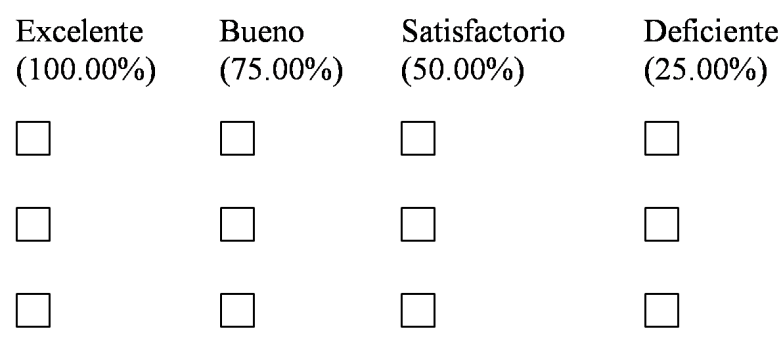

(31.82\%) Contiene herramientas de acceso y actividad

Excelente Bueno Satisfactorio Deficiente

$(100.00 \%) \quad(75.00 \%) \quad(50.00 \%) \quad(25.00 \%)$

(22.73\%) Cuenta con herramientas par acceder a información o contiene información (ejem:mediatecas)

(22.73\%) Cuenta con herramientas donde comente, analice y publique ( ejem:bolgs)

$(22.73 \%)$ Cuenta con herramientas donde se relacione con otros ( ejem: red social)

\section{(28.00\%) Creatividad en el PLE}

(44.44\%) Originalidad del contenido

Excelente Bueno Satisfactorio Deficiente $(100.00 \%)(75.00 \%)(50.00 \%) \quad(25.00 \%)$

(33.33\%) Presencia de Ideas Inusuales

(33.33\%) Su contenido no es banal, ni obvio

(33.33\%) Su contenido no es similar a otros PLE

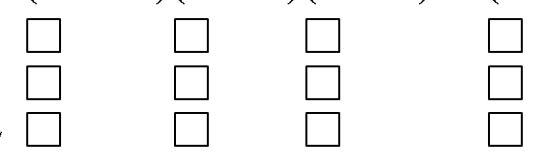

(33.33\%) Fluidez del contenido

(33.33\%) El contenido incentiva a su exploración Excelente Bueno Satisfactorio Deficiente $(100.00 \%)(75.00 \%)(50.00 \%) \quad(25.00 \%)$

$(33.33 \%)$ Existe una relación coherente entre los contenidos 